Article

\title{
Investigation of Acoustic Properties on Wideband Sound-Absorber Composed of Hollow Perforated Spherical Structure with Extended Tubes and Porous Materials
}

\author{
Dengke Li ${ }^{1,2, *}$, Zhongcheng Jiang ${ }^{2}$, Lin $\mathrm{Li}^{3}{ }^{3}$, Xiaobo Liu ${ }^{2}$, Xianfeng Wang ${ }^{2}$ and $\mathrm{Mu} \mathrm{He}^{4}$ \\ 1 Train and Track Research Institute, State Key Laboratory of Traction Power, Southwest Jiaotong University, \\ Chengdu 610031, China \\ 2 The State Key Laboratory of Heavy Duty AC Drive Electric Locomotive Systems Integration, \\ Zhuzhou 412001, China; Jiangzhongcheng.zz@crrcgc.cc (Z.J.); Liuxiaobo.zz@crrcgc.cc (X.L.); \\ Wangxianfeng.zz@crrcgc.cc (X.W.) \\ 3 National Innovation Center of Advanced Rail Transit Equipment, Zhuzhou 412001, China; Lilin.zz@crrcgc.cc \\ 4 Green Building, Energy Saving and Noise Reduction Research Center, Department of Urban Construction, \\ City College, Wuhan University of Science and Technology, Wuhan 430083, China; mu.he@wic.edu.cn \\ * Correspondence: ldk@mail.ioa.ac.cn or lidengke.zz@crrcgc.cc
}

Received: 4 November 2020; Accepted: 11 December 2020; Published: 16 December 2020

\begin{abstract}
Traditional porous media such as melamine foam absorb sound due to their three-dimensional porous struts. However, the acoustic properties at low frequencies are greatly related to its thickness. In this paper, a novel type of thin and lightweight sound absorber composed of melamine foam and hollow perforated spherical structure with extended tubes (HPSET) is introduced to enhance the sound absorption performance at low frequencies. A theoretical model for the normal absorption coefficient of the HPSET with melamine foam is established. Good agreements are observed between the simulated and the experimental results. Compared with the virgin melamine foam, the proposed absorber can greatly improve the low-frequency sound absorption and retain the mid- to high-frequency sound absorption, while the thickness of the proposed absorber is less than $1 / 28$ of the wavelength.
\end{abstract}

Keywords: hollow perforated spherical structure with extended tubes; low frequency sound absorption; melamine foam; wideband sound absorber

\section{Introduction}

Melamine foams are porous materials widely used in the transport and civil engineering industries for their remarkable properties of sound absorption and special abilities to withstand extreme environments (such as heat insulation, fire protection and environmental protection). At present, the research on this type of foam material has produced a series of papers, and the manufacturing process is protected by a large family of patents [1,2]. However, in practical noise applications, such as rail locomotive vehicles, the internal noise of the vehicle is mainly dominant in the low-frequency noise of 100-1000 Hz. If a single layer of melamine foam is used to absorb the low frequency noise inside the cab, the foam materials usually require a relatively large space and material thickness [3]. Many researches focused on the optimization of the pore size of porous foams, since the sound energy dissipation mechanism of porous materials originates from the visco-thermal dissipation of micropores. Perrot [4] studied the sound absorption properties of the open-cell foam metal structure based on the Kelvin structure. He pointed out that the pore size directly determines the flow resistivity of the 
material. When the pore size is small, the flow resistivity of the material increases, and the sound wave is not easy to enter the material; when the pore structure is large, the flow resistivity is very small, and the large-size micropores cannot provide sufficient damping for the incident sound waves. Later, Trinhet et al. [5] studied the sound absorption of polyurethane foam with membrane in the pore network, and their results show that decreasing the openness of the membrane could enhance sound absorption performances of the material in low frequency ranges. Park et al. [6,7] built a multiscale numerical model to optimize the sound absorption properties of PU foams, they found that the acoustic damping at low frequencies could be improved with an optimum mean cell size and cell openness.

Optimization of the geometrical parameters of the porous structure could improve the sound absorption of the porous foams to some extent; however, their first sound absorption peak frequency is still determined by its quarter wavelength resonance frequency. In recent years, much attention has been paid on developing meta-acoustic materials to enhance the low frequency absorbing performance. Kidner and Fuller experimentally investigated the use of heterogeneous (HG) acoustic materials to improve low frequency insertion loss of blankets [8]. An active-passive method, which, based on FOAM-PVDF structure, was also introduced by Fuller to enhance the transmission loss of foam materials [9]. Fuller and his colleagues further [10] used meta-materials that are composed of small masses and poro-elastic media to improve the sound absorption of the porous materials at low frequencies. Based on numerical analyses of the finite element method, Groby et al. [11,12] conducted several studies on periodic inclusions embedded in the porous layer to improve the sound absorption bandwidth. However, their effective sound absorption bandwidth of the composite absorber still lies in the mid-or high-frequency range $(>1000 \mathrm{~Hz})$.

Relying on the multi-layer resonance system, the sound absorption bandwidth of porous media could be significantly broadened by using the perforated plates. Lin [13] studied the structure of the sound-absorbing material behind the micro-perforated plate, and their results revealed that, when the sound-absorbing material occupied the entire cavity, the combined structure had a broader sound absorption band. Li et al. [14] studied theoretically and experimentally the sound absorption coefficient of sound-absorbing materials combined with micro-perforated plates by using the transfer matrix method. They analyzed influences of different placement of sound-absorbing materials on the sound absorptive performance for the composite absorber, and proposed a wideband sound-absorbing configuration in which the sound-absorbing material was placed in front of the micro-perforated plate. However, the composite absorber still requires a large installation space.

More recent work has laid foundations of improving the low frequency sound absorption performance by using extended tube resonators [15-19]. Li et al. [16-18] presented a kind of multiple extended tube resonators to enhance the low frequency range from 100 to $1600 \mathrm{~Hz}$ in a constrained space of $100 \mathrm{~mm}$. In order to further improve the low frequency sound absorption of a thin layer melamine foam below $500 \mathrm{~Hz}$, a new type of resonant absorber comprised of hollow perforated spherical structure with extended tubes is introduced in the present work. Hence we theoretically and experimentally investigated the low sound absorption performance of the combined absorber, and found that the coupling between the Helmholtz resonance and the quarter wavelength resonance shows a great potential to ameliorate sound absorptive performance of a traditional porous foam at low- and mid-frequencies. Meanwhile, the sound absorption in low frequency range could be greatly enhanced by tuning the tube parameters. Our proposed sound absorber reaches the same sound absorption performance of PPETs-PSAM [17,18], while it is more practical in noise control application since this device is simply made of a thin and lightweight hollow perforated spherical structure, and could be easily combined with porous foams. In what follows, we firstly conduct a theoretical analysis of the performance of combined absorber in Section 2, and then focus on parametric studies in Section 3. Section 4 is aimed at experimental verifications by impedance tube. Finally, Section 5 draws some conclusions. 


\section{Theoretical Analyses}

\subsection{Impedance Model of Melamine Foam}

Following the well-known JCAL model proposed by Johnson et al. [20] and Lafarge et al. [21], the equivalent density $\rho_{e q}(\omega)$ and modulus $K_{e q}(\omega)$ of the porous fluid are

$$
\begin{gathered}
\rho_{e q}(\omega)=\frac{\alpha_{\infty} \rho_{0}}{\phi}\left[1-j \frac{\sigma \phi}{\omega \rho_{0} \alpha_{\infty}} \sqrt{1+j \frac{4 \alpha_{\infty}^{2} \eta \rho_{0} \omega}{\sigma^{2} \Lambda^{2} \phi^{2}}}\right] \\
K_{e q}(\omega)=\frac{\gamma \rho_{0} / \phi}{\gamma-(\gamma-1)\left[1-j \frac{\phi \eta}{k_{0^{\prime} N_{p r} \rho_{0} \omega}} \sqrt{1+j \frac{4 k_{0^{\prime}{ }^{\prime 2} N_{p r} \rho_{0} \omega}^{\eta \Lambda^{\prime 2} \phi^{2}}}{-1}}\right.}
\end{gathered}
$$

where $\omega$ is the angular frequency, $\rho_{0}$ is the density of the air, $\eta$ is the viscosity of the air, $\sigma$ is the airflow resistivity, $P_{0}$ is the mean ambient pressure, $\phi$ is the porosity of the material considered, $N_{p r}$ is the Prandtl number of the air, and $\gamma=C_{p} / C_{v}$ is the specific heat ratio, in which $C_{p}$ and $C_{v}$ are the specific heat capacities at constant pressure and at constant volume respectively. The JCAL model involves six characteristic parameters: the static viscous permeability $k_{0}$, the porosity $\phi$, the tortuosity $\alpha_{\infty}$, the viscous characteristic length $\Lambda$, the static thermal permeability $k_{0}{ }^{\prime}$ and the thermal characteristic length $\Lambda^{\prime}$.

According to formula (1) and formula (2), the wave number $k_{s}$ and characteristic impedance $Z_{s}$ of the equivalent fluid medium is

$$
\begin{gathered}
Z_{s}(\omega)=\sqrt{\rho_{e q}(\omega) K_{e q}(\omega)}, \text { and } \\
k_{s}(\omega)=\omega \sqrt{\rho_{e q}(\omega) / K_{e q}(\omega)}
\end{gathered}
$$

For the melamine foam with a thickness of $H$, the surface impedance at $x=H$ of the sample backed by a rigid wall (see Figure 1) is given by

$$
Z_{P M}(\omega)=\frac{-j Z_{s}(\omega) \cot \left(k_{s}(\omega) H\right)}{\phi}
$$

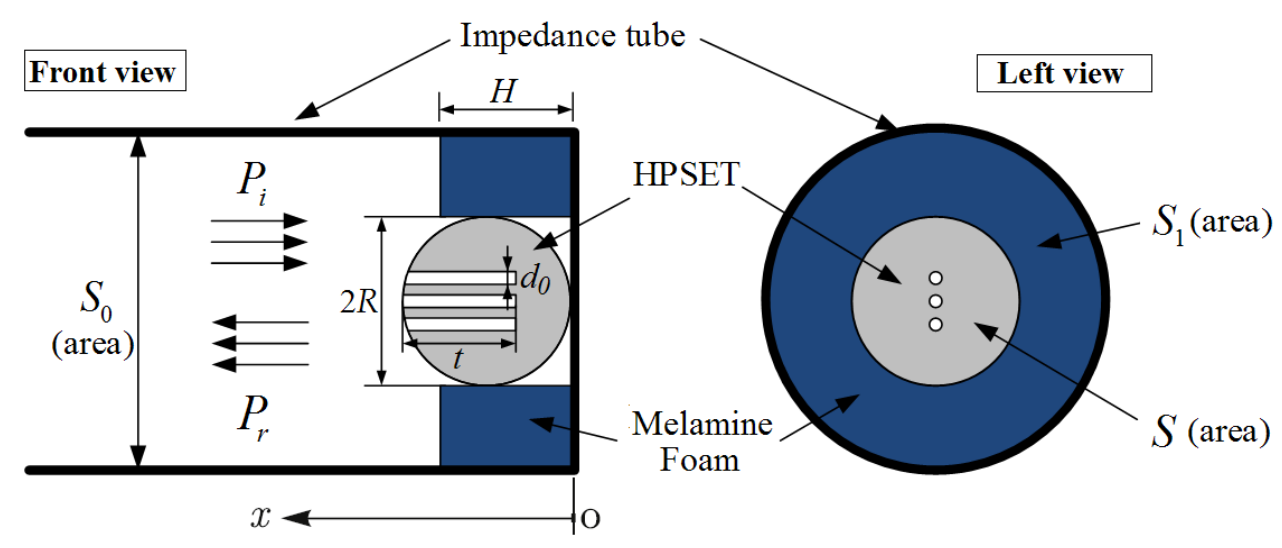

Figure 1. Illustration of HPSET and melamine foam installed at an impedance tube. The thickness of the foam is $H$, and the inner diameter of the HPSET is $2 R$. The diameters and the maximum length of the extended tubes are $d_{0}$ and $t$. All extended tubes inlets are shaped to follow the sphere curvature. The cross-sectional areas of HPSET and impedance tube are $S$ and $S_{0}$. 
In this paper, an experimental characterization approach, which requires direct measurements of $\phi$ and $k_{0}$ and an impedance tube technique [22-24], is adopted to characterize the transport parameters of melamine foam $[25,26]$. Parameters of the melamine foam are listed in Table 1 . The porosity $\phi$ is measured by a porosimeter, the air-flow resistivity $\sigma$ is directly measured by resistivimeter, and the remaining four transport parameters $\left(\alpha_{\infty}, \Lambda, \Lambda^{\prime}, k_{0}{ }^{\prime}\right)$ are determined by the inverse characterization techniques using a three-microphone impedance tube [24]. We use a commercially available software RokCell (v3.0, MATELYS, Lyon, France) [27] to automatically obtain the estimations of the parameters.

Table 1. Parameters of the melamine foam.

\begin{tabular}{ccccccc}
\hline Parameters & $\boldsymbol{\sigma}\left(\mathbf{P a} \cdot \mathbf{s} / \mathbf{m}^{2}\right)$ & $\boldsymbol{\phi}$ & $\boldsymbol{\alpha}_{\infty}$ & $\boldsymbol{\Lambda}(\boldsymbol{\mu m})$ & $\Lambda^{\prime}(\mu \mathrm{m})$ & $k_{0}{ }^{\prime}\left(\times 10^{-10} \mathbf{m}^{2}\right)$ \\
\hline $\begin{array}{c}\text { Melamine } \\
\text { foam }\end{array}$ & $9354 \pm 390$ & $0.993 \pm 0.001$ & $1.04 \pm 0.02$ & $107 \pm 5$ & $223 \pm 23$ & $23 \pm 2$ \\
\hline
\end{tabular}

\subsection{Impedance Model of the Hollow Perforated Spherical Structure with Extended Tubes (HPSET)}

Figure 1 illustrates the placement of the HPSET and melamine foam in an impedance tube. According to the well-known Maa model [28,29], the acoustic impedance of a single extended tube can be expressed as

$$
Z=\frac{\Delta P}{\bar{u}}=j \omega \rho_{0}\left(t+0.85 d_{0}\right)\left[1-\frac{2}{x \sqrt{-j}} \frac{J_{1}(x \sqrt{-j})}{J_{0}(x \sqrt{-j})}\right]^{-1}+\frac{\sqrt{2 \omega \rho_{0} \eta}}{4}
$$

where $x=d_{0} \sqrt{\omega \rho_{0} /(4 \eta)}$ is the ratio between the perforation radius and the viscous boundary layer thickness inside the tube of the perforations (also named "perforation constant"), $d_{0}$ is the inner diameter of the extended tubes, $t$ is the maximum length of the tubes, $\eta$ is the viscosity of the air, $\omega$ denotes the angular frequency, $j=\sqrt{-1}$ represents the imaginary unit, $\rho_{0}$ is the mass density of the air, and $J_{0}, J_{1}$ are Bessel's functions of zero and first order.

The normalized impedance of the spherical resonating cavity is $Z_{D}=-j \cot \left(\frac{\omega}{c} \frac{\left(V-V_{\text {tubes }}\right.}{S}\right)$, then the normalized impedance of HPSET absorber is given as

$$
Z_{\text {HPSET }}=\frac{Z}{\varphi_{p} \rho_{0} c}+Z_{D}=r_{p}+j \omega m_{p}-j \cot \left(\frac{\omega}{c} \frac{\left(V-V_{\text {tubes }}\right)}{S}\right)
$$

with

$$
\begin{gathered}
r_{p}=\frac{32 \eta t}{\varphi_{p} \rho_{0} c d_{0}^{2}}\left(\left(1+\frac{x^{2}}{32}\right)^{1 / 2}+\frac{\sqrt{2} x d_{0}}{64 t}\right) \\
\omega m_{p}=\frac{\omega t}{\varphi_{p} c}\left(1+\left(9+\frac{x^{2}}{2}\right)^{-1 / 2}+0.85 \frac{d_{0}}{t}\right)
\end{gathered}
$$

where $\rho_{0} c$ denotes the characteristic impedance of the air, $c$ is the sound speed in the air $(\mathrm{m} / \mathrm{s})$, $\phi_{p}=N A_{0} / S$ corresponds to the perforation ratio of the HPSET ( $N$ denotes the number of extended tubes, $A_{0}=\pi d_{0}^{2} / 4$ denotes to the inner cross-sectional area of the extended tubes, $S=\pi R^{2}$ is the cross-sectional area of the HPSET. $d_{0}$ is the inner diameter of the extension tubes and $R$ is the inner radius of the HPSET). $V=4 \pi R^{3} / 3$ and $V_{\text {tubes }}=\pi t N d_{0}{ }^{2} / 4$ are the volume of the perforated ball and the extended tubes, respectively.

\subsection{Normal Incidence Sound Absorption of HPSET with a Melamine Foam}

Considering the sound waves impinges vertically on the composite absorber as illustrated in Figure 1. As the HPSET is embedded within the melamine foam, the equivalent impedance is the 
parallel of the HPSET and the melamine. Based on the impedance transfer formula [30], the specific acoustic impedance of the melamine foam at the surface of the composite absorber can be calculated as

$$
Z_{P M}^{\prime}=\frac{Z_{P M}(\omega)+j \rho_{0} c \tan \left(k_{s}(\omega)(R-H)\right)}{\rho_{0} c+j Z_{P M}(\omega) \tan \left(k_{s}(\omega)(R-H)\right)}
$$

Then, the characteristic impedance of the composed absorber is given as

$$
Z^{\prime}=\left(\frac{1-\varphi_{b}}{Z_{P M}^{\prime}}+\frac{\varphi_{b}}{Z_{H P S E T}}\right)
$$

where $\varphi_{b}=S / S_{0}$, and $S_{0}$ denotes the cross-sectional area of the impedance tube, $S$ denotes the cross-sectional area of the HPEST. The normal incidence sound absorption coefficient of HPSET combined with melamine foam installed at an impedance tube is calculated as

$$
\alpha=\frac{4 \operatorname{Real}\left(Z^{\prime}\right)}{\left(1+\operatorname{Real}\left(Z^{\prime}\right)^{2}\right)+\operatorname{Imag}\left(Z^{\prime}\right)^{2}}
$$

\section{Simulation Results and Discussion}

\subsection{Analytical Study of Normal Incidence Sound Absorption Coefficient of the HPSET with a Melamine Foam}

Figure 2 illustrates the analytical results of the normal incidence sound absorption coefficient of the HPSET with a melamine foam obtained from Equation (12), in which the sound absorption of HPSET and a single layer melamine foam are also shown for a comparison. In the following simulations, the inner diameter of the HPSET is $2 R=65 \mathrm{~mm}$, the number of perforations is $N=2$, the diameters and lengths of the extended tubes are $d_{0}=4.9 \mathrm{~mm}$ and $t=10 \mathrm{~mm}$, respectively. The Helmholtz resonance absorption peak of the HPSET is found at $380 \mathrm{~Hz}$ and the anti-resonance frequency of this combined absorber is observed at $500 \mathrm{~Hz}$, while the quarter wavelength resonance frequency of the porous material is around $1500 \mathrm{~Hz}$. It is shown in Figure 2a that the sound absorption of the composite absorber is superior to the single layer melamine foam and HPSET. It is clear that the sound absorption of a single layer porous foam is less than 0.5 below $500 \mathrm{~Hz}$. While the composite absorber reaches a wideband sound absorption (greater than 0.5) in the frequency range from $350 \mathrm{~Hz}$ to $2000 \mathrm{~Hz}$, hence combination of HPSET with melamine foam could greatly enhance the low frequency sound absorptive performance of the melamine foam.

It is revealed from Figure $2 b$ that the relative resistance of single layer melamine foam and HPSET are less than 1, which is less than the combined absorber in the frequency range from $360 \mathrm{~Hz}$ to $2000 \mathrm{~Hz}$. Hence, the present absorber could improve the acoustic resistance and enhance the sound absorption. Meanwhile, the relative acoustic reactance of this combination is nearly zero at the resonance frequency of $380 \mathrm{~Hz}$ and $1500 \mathrm{~Hz}$, which ensures the wideband absorptive performance of the proposed absorber. The low frequency sound absorptive performance could be further enhanced by combining multiple HPSETs with different resonance frequency.

\subsection{Parametric Study of the Sound Absorption Coefficient of HPSET with a Melamine Foam}

Since the sound absorption of HPSET-Melamine foam absorber is influenced by many parameters, we take the same analytical process and investigate the main parameters in this section. The resistivity of the melamine foam is a key factor which dominates the sound absorption. It is demonstrated in Figure $3 \mathrm{a}$ that the resistivity of the melamine foam will greatly influence sound absorption both at the anti-resonance frequency and the quarter wavelength resonance frequency. When increasing the resistivity of the melamine foam, the sound absorption at the anti-resonance frequency is enhanced, while the sound absorption at the quarter wavelength resonance frequency is firstly increased and then decreased. The absorption peak at Helmholtz resonance frequency decreases slightly with the 
increase in resistivity. Hence, a reasonable resistivity is required to match the specific resistance of the incident sound waves for the composite absorber.

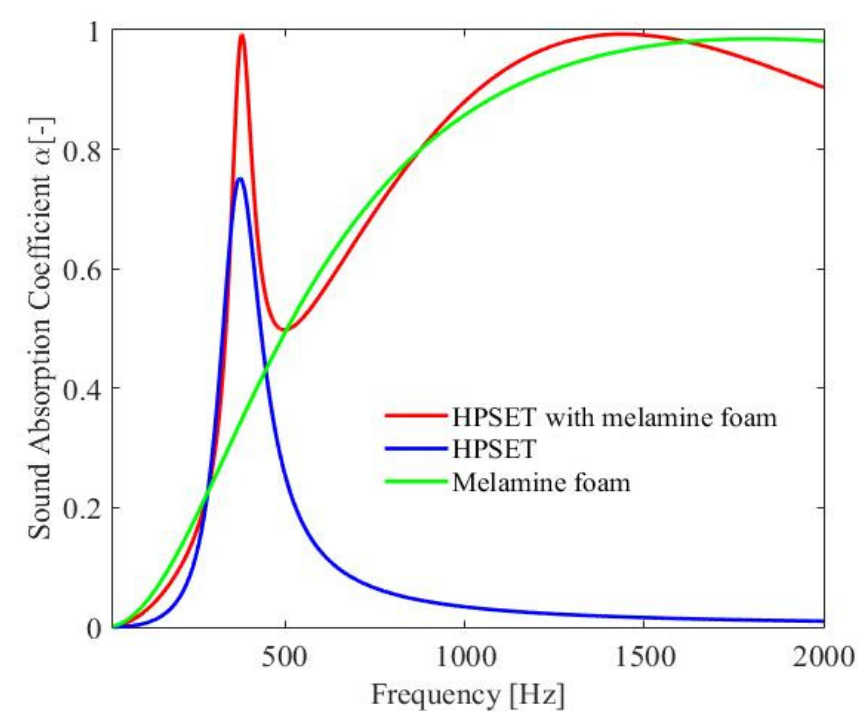

(a)

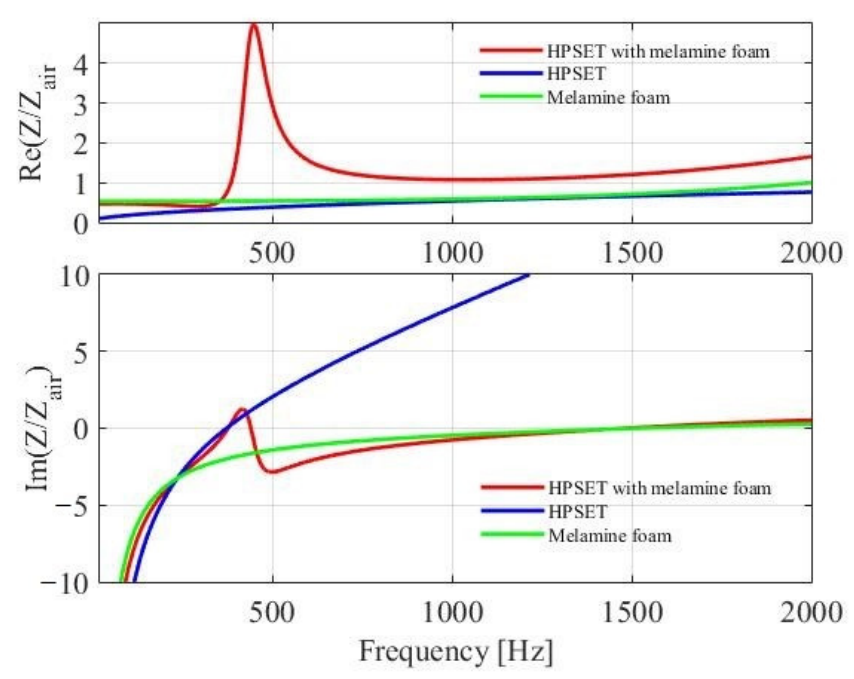

(b)

Figure 2. Analytical results of normal incidence sound absorption of HPSET with melamine foam. (a) Normal incidence sound absorption coefficient; (b) Characteristic acoustic resistance and reactance.

Figure $3 \mathrm{~b}$ demonstrates the variation of the diameter of extended tubes on the overall sound absorption of the present absorber. The tube diameter is a critical factor which controls the sound absorption performance of the sound absorption of HPSET. When the tube diameter is too large, the relative resistance is less than 1, and the sound absorption is decreased. On the opposite, a small-diameter will induce overlarge acoustic resistance which will also decrease the sound absorption.

As illustrated in Figure 3c,d, the low frequency sound absorption peak of the combined absorber is greatly shifted to lower frequencies by decreasing the tube number or increasing the tube length, while the high sound absorption peak remains the same. It is noted that the low frequency sound absorption of the HPSET is due to the increase in mass reactance, and the resonance frequency could be tuned via optimizing the tube parameters. 


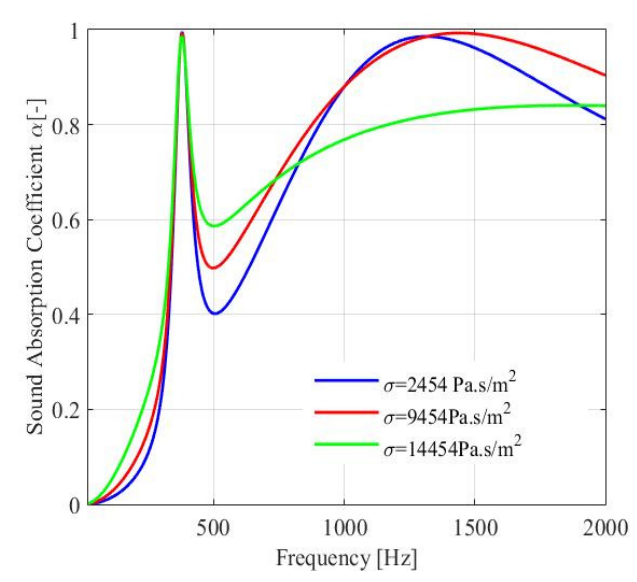

(a)

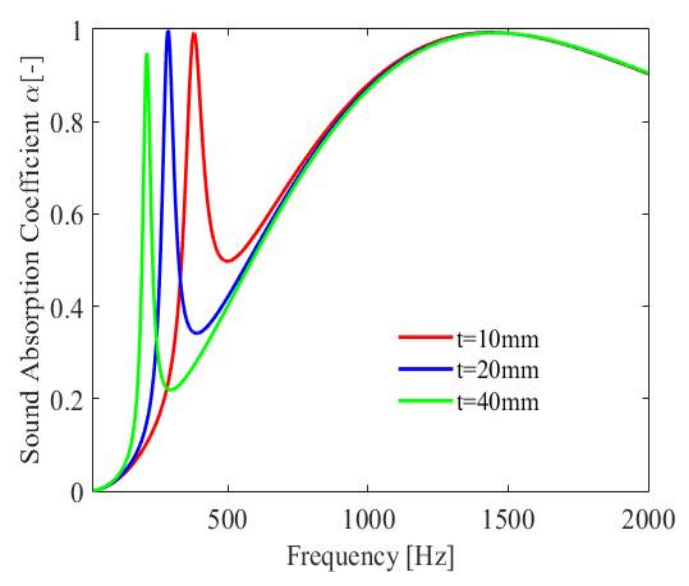

(c)

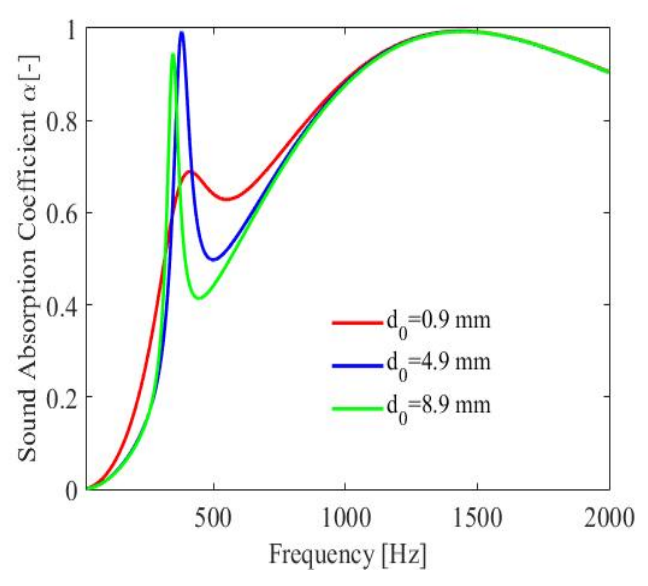

(b)

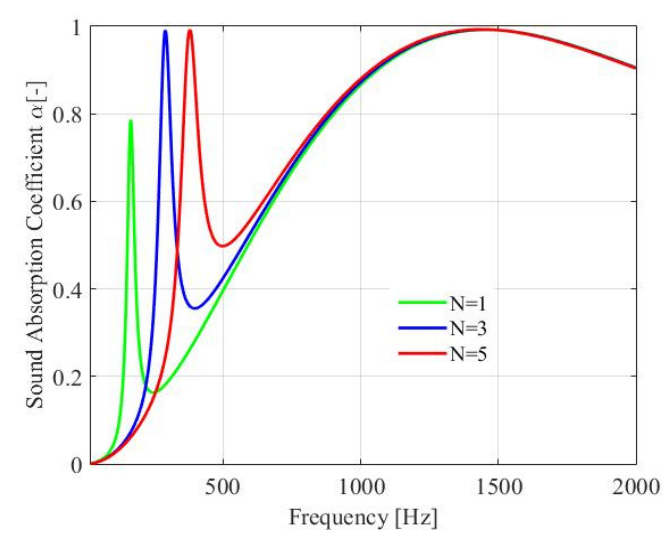

(d)

Figure 3. Comparison of the normal incidence sound absorption of composite absorber with different parameters. (a) The resistivity of melamine foam; (b) The diameter of extended tubes; (c) The length of extended tubes; (d) The number of extended tubes.

\section{Experimental Validation}

The normal-incidence sound absorption coefficient of the HPSET with melamine foam is measured by an impedance tube (B\&K 4206, see Figure 4) based on the transfer function method. The measurements are manipulated according to the ISO 10534-2 standard [31], and the experiment set up is shown in Figure 4. The distance between the two microphones is $50 \mathrm{~mm}$ and the measured frequency range is from 0 to $1600 \mathrm{~Hz}$. The inner diameter of the impedance tube is $100 \mathrm{~mm}$. The room temperature is $17.5^{\circ} \mathrm{C}$, the atmosphere pressure is $1.01 \times 10^{5} \mathrm{~Pa}$, and the relative humidity is $66.4 \%$. The hollow perforated spherical structure used in the experiments is made of plastic, and the extended tubes are made of copper. Parameters for the HPSETs are shown in Table 2. Thickness of hollow perforated spherical structure and extended tubes is $0.5 \mathrm{~mm}$. The thickness of the foam is $50 \mathrm{~mm}$, and the average densities of the HPSET with foam are $47.1 \mathrm{~kg} / \mathrm{m}^{3}$.

Table 2. Parameters of the tested samples of HPSETs.

\begin{tabular}{ccccc}
\hline Parameters & $\boldsymbol{d}_{\mathbf{0}}(\mathbf{m m})$ & $\boldsymbol{t}(\mathbf{m m})$ & $\boldsymbol{N}$ & $\mathbf{2 R}(\mathbf{m m})$ \\
\hline HPSET $_{1}$ & 4.9 & 10 & 2 & 65 \\
HPSET $_{2}$ & 4.9 & 10 & 5 & 65 \\
\hline
\end{tabular}


Figure 5 shows the comparison of the experimental result and the calculation result for the sound absorption coefficient of the melamine foam. The calculation result uses the inverse characterization techniques described in Section 2.1 (inversion method-based parameters are listed in Table 1). It is shown that the calculation result is highly consistent with the actual experimental result, which verifies the reliability of this inversion characterization method.

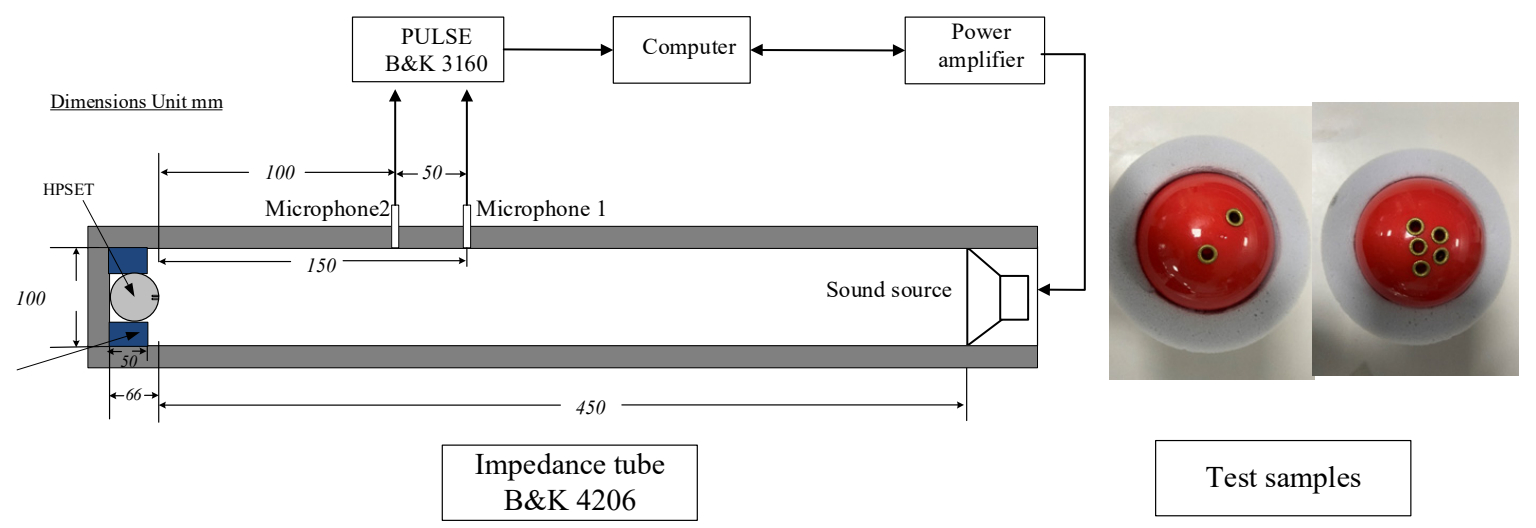

Figure 4. Experiment set up.

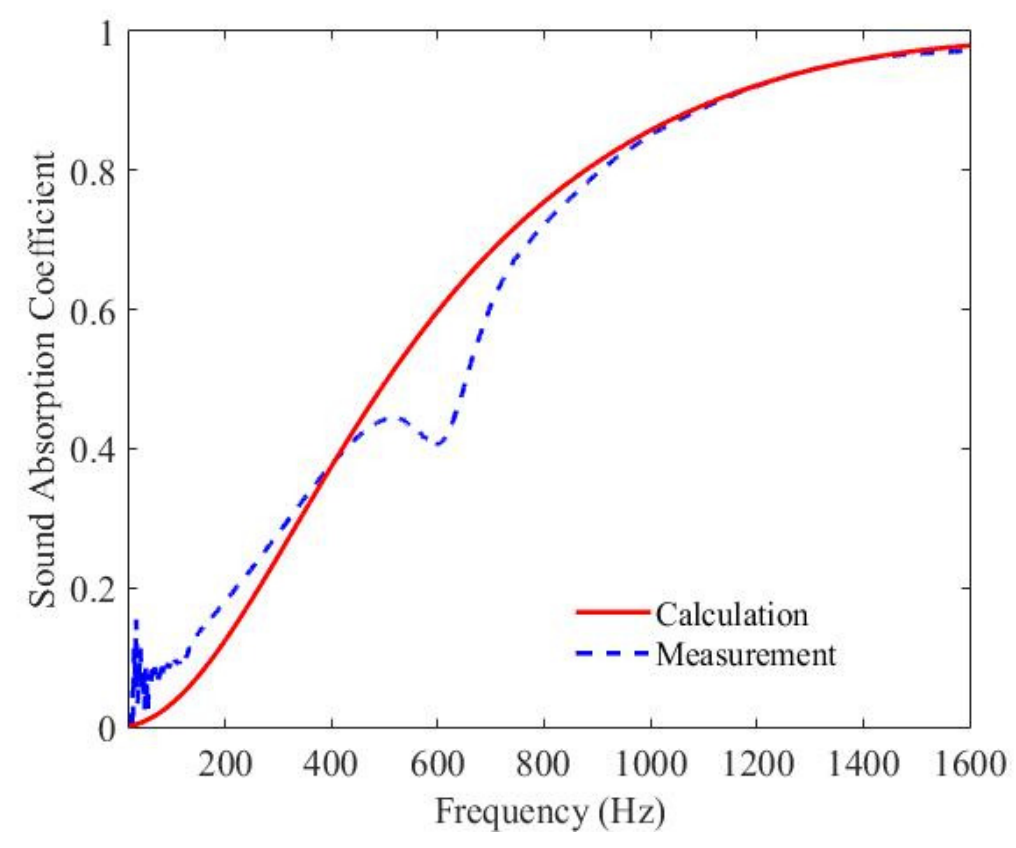

Figure 5. Sound absorption coefficient of the melamine foam. The red solid line and blue dashed line represent the calculated results by inversion method and directly measured results, respectively.

The measured and calculated sound absorption coefficient curves of HPSET with melamine foam are shown in Figure 6. Good agreement is observed between the measurement and calculation. The HPSET combined with a porous foam in a limited thickness of $65 \mathrm{~mm}$ reaches a good sound absorption property in the frequency range from 200 to $1600 \mathrm{~Hz}$. The thickness of the porous material is only $1 / 28$ of the sound wavelength, which realizes the purpose of controlling the large wavelength with thin layer materials. 


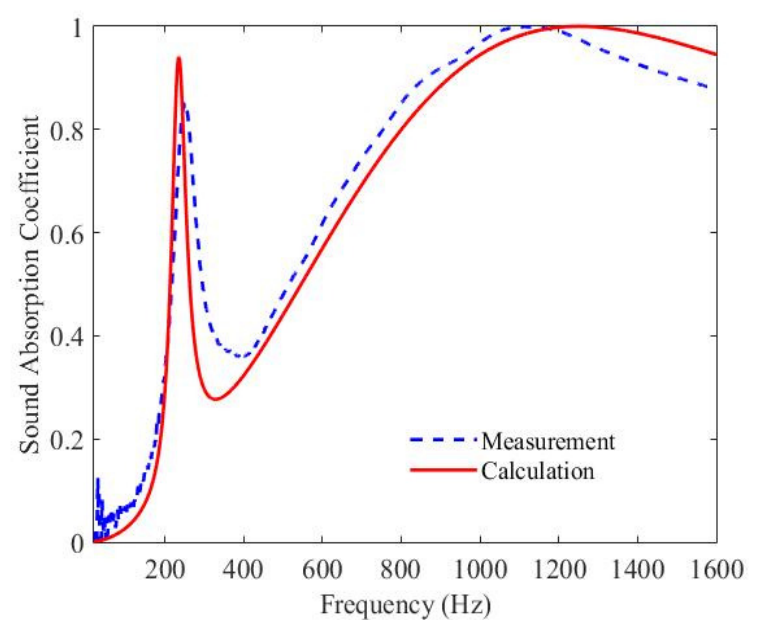

(a)

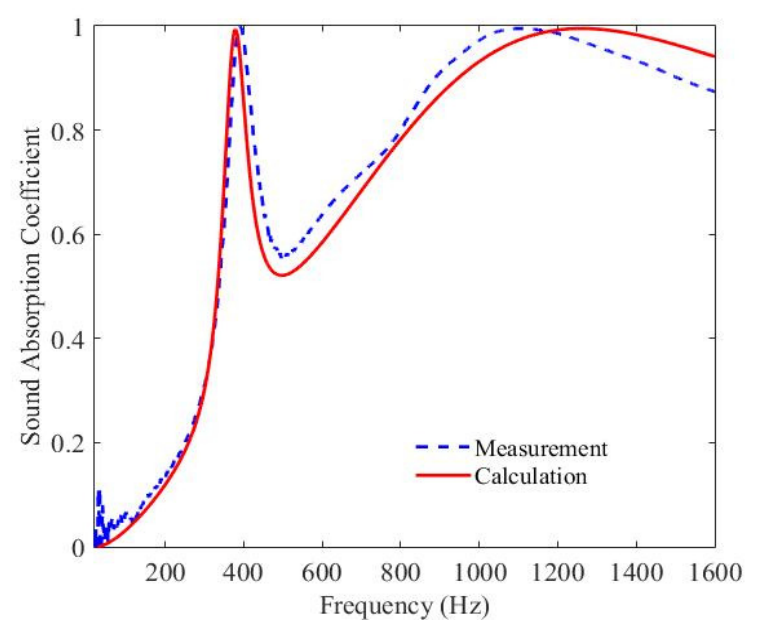

(b)

Figure 6. Sound absorption coefficient of the composite absorber for (a) $\mathrm{HPSET}_{1}$ and (b) $\mathrm{HPSET}_{2}$ (see Table 2). Red solid line: calculation by inversion method; Blue dashed line: direct measurement.

\section{Conclusions}

A thin and lightweight sound absorber is presented to improve the acoustic properties at low frequencies of melamine foams. The absorption performance of the compound absorber is validated experimentally by using an impedance tube, and the measured results are consistent with the calculation results. Our research implies that the tube diameter and the resistivity are critical factors controlling the absorption performance. When decreasing the tube number or increasing the tube length, the resonance frequency is greatly shifted to lower frequencies. Both theoretical and measured results show that the HPSET combined with a melamine foam can keep good sound absorptive performance in the frequency range from $200-1600 \mathrm{~Hz}$ in a limited thickness. Compared with conventional absorbers, the proposed absorber is practical in noise control applications such as in domains of rail vehicles, aircrafts cabin and automobiles.

Author Contributions: Conceptualization, L.L.; Methodology, D.L.; Software, M.H.; Formal Analysis, L.L. and Z.J.; Investigation, D.L. and M.H.; Data Curation, X.L. and X.W.; Writing-Original Draft Preparation, D.L.; Writing-Review and Editing, M.H. and Z.J.; Visualization, L.L. and D.L. All authors have read and agreed to the published version of the manuscript.

Funding: The first author would like to acknowledge the support from the project of China Postdoctoral Science Foundation (Grant No. 256069).

Conflicts of Interest: The authors declare no conflict of interest. 


\section{References}

1. Imashiro, Y.; Hasegawa, S.; Matsumoto, T. Melamine Resin Foam. U.S. Patent No. 5,413,853, 9 May 1995.

2. Thom, A. Melamine Resin Foam. U.S. Patent No. 6,350,511, 26 February 2002.

3. Fuchs, H. Alternative fibreless absorbers-New tools and materials for noise control and acoustic comfort. Acta. Acoust. 2001, 87, 414-422.

4. Perrot, C.; Chevillotte, F.; Panneton, R. Dynamic viscous permeability of an open-cell aluminum foam: Computations vs experiments. J. Appl. Phys. 2008, 103, 024909. [CrossRef]

5. Trinh, V.H.; Hoang, M.T.; Perrot, C.; Langlois, V.; Khidas, Y.; Pitois, O. A systematic link between microstructure and acoustic properties of foam: A detailed study on the effects of membranes. In Proceedings of the 6th Biot Conference of Poromechanics, Paris, France, 9-13 June 2017.

6. Park, J.H.; Minn, K.S.; Lee, H.R.; Yang, S.H.; Yu, C.B.; Pak, S.Y.; Oh, C.S.; Song, Y.S.; Kang, Y.J.; Youn, J.R. Cell openness manipulation of low density polyurethane foam for efficient sound absorption. J. Sound Vib. 2017, 406, 224-236. [CrossRef]

7. Park, J.H.; Yang, S.H.; Lee, H.R.; Yu, C.B.; Pak, S.Y.; Oh, C.S.; Kang, Y.J.; Youn, J.R. Optimization of low frequency sound absorption by cell size control and multiscale poroacoustics modeling. J. Sound Vib. 2017, 397, 17-30. [CrossRef]

8. Kidner, M.; Gardner, B.; Fuller, C. Improvements in Low Frequency Insertion Loss Blankets: Experimental Investigation. J. Sound Vib. 2006, 294, 466-472. [CrossRef]

9. Fuller, C.; Kidner, M.; Li, X.; Hansen, C. Active-Passive Heterogeneous for Control of Vibration and Sound Radiation. In Proceedings of the 2004 International Symposium on Active Control of Sound and Vibration, Williamsburg, VA, USA, 20-22 September 2004.

10. Fuller, C. Sound absorption using acoustic meta materials. In Proceedings of the Inter-Noise 2012, New York, NY, USA, 19-22 August 2012.

11. Groby, J.P.; Lagarrigue, C.; Brouard, B.; Dazel, O.; Tournat, V.; Nennig, B. Enhancing the absorption properties of acoustic porous plates by periodically embedding Helmholtz resonators. J. Acout. Soc. Am. 2015, 137, 273-280. [CrossRef] [PubMed]

12. Groby, J.P.; Lagarrigue, C.; Brouard, B.; Dazel, O.; Tournat, V.; Nennig, B. Using simple shape three-dimensional rigid inclusions to enhance porous layer absorption. J. Acout. Soc. Am. 2014, 136, 1139-1148. [CrossRef] [PubMed]

13. Lin, L.; Wang, Z.; Jiang, Z. Effect of sound-absorbing material on a micro-perforated absorbing construction. Acta. Acust. 2010, 35, 385-391.

14. Li, D.; Chang, D.; Liu, B.; Tian, J. Improving the sound absorption bandwidth of micro-perforated panel by adding porous sound absorbing materials. In Proceedings of the Internoise 2014, Melbourne, Australia, 16-19 November 2014.

15. Lu, Y.; Li, X.; Tian, J.; Wei, W. The perforated panel resonator with flexible tube bundle and its acoustical measurements. In Proceedings of the Internoise 2001, The Hague, The Netherlands, 27-30 August 2001.

16. Li, D.; Chang, D.; Liu, B. Enhancing the low frequency sound absorption of a perforated panel by parallel-arranged extended tubes. Appl. Acoust. 2016, 102, 126-132. [CrossRef]

17. Li, D.; Chang, D.; Liu, B. Enhanced low- to mid-frequency sound absorption using parallel-arranged perforated plates with extended tubes and porous material. Appl. Acoust. 2017, 127, 316-323. [CrossRef]

18. Li, D.; Chang, D.; Liu, B. Diffuse sound absorptive properties of parallel-arranged perforated plates with extended tubes and porous materials. Materials 2020, 13, 1091. [CrossRef]

19. Simon, F. Long Elastic Open Neck Acoustic Resonator for low frequency absorption. J. Sound Vib. 2018, 421, 1-16. [CrossRef]

20. Johnson, D.L.; Koplik, J.; Dashen, R. Theory of dynamic permeability and tortuosity in fluid-saturated porous media. J. Fluid Mech. 1987, 176, 379-402. [CrossRef]

21. Lafarge, D.; Lemarinier, P.; Allard, J.F.; Tarnow, V. Dynamic compressibility of air in porous structures and audible frequencies. J. Acout. Soc. Am. 1997, 102, 1995-2006. [CrossRef]

22. Stinson, R.; Daigle, G. Electronic system for the measurement of flow resistance. J. Acout. Soc. Am. 1988, 83, 2422-2428. [CrossRef] 
23. Iwase, T.; Izumi, Y.; Kawabata, R. A New Measuring Method for Sound Propagation by Using Sound Tube without Any Air Spaces. In Proceedings of the 1998 International Congress on Noise Control Engineering, Christchurch, New Zealand, 16-18 November 1998.

24. Salissou, Y.; Panneton, R. Wideband characterization of the complex wave and characteristic impedance of sound absorbers. J. Acout. Soc. Am. 2010, 128, 2868-2876. [CrossRef] [PubMed]

25. Panneton, R.; Olny, X. Acoustical determination of the governing viscous dissipation in porous media. J. Acout. Soc. Am. 2006, 119, 2027-2040. [CrossRef]

26. Olny, X.; Panneton, R. Acoustical determination of the governing thermal dissipation in porous media. J. Acout. Soc. Am. 2008, 123, 814-824. [CrossRef]

27. Available online: https://rokcell.matelys.com/ (accessed on 4 December 2020).

28. Maa, D.Y. Theory and design of micro-perforated sound absorbing constructions. Sci. Sin. 1975, 18, 55-71.

29. Maa, D.Y. Microperforated-panel wideband absorbers. Noise Control Eng. J. 1987, 29, 77-84. [CrossRef]

30. Allard, J.; Atalla, N. Propagation of Sound in Porous Media: Modelling Sound Absorbing Materials, 2nd ed.; Wiley and Sons: London, UK, 2009; Chapter 2.

31. ISO 10524-2. Acoustics—Determination of Sound Absorption Coefficient and Impedance in Impedance Tubes-Part 2: Transfer Function Method; ISO: Geneva, Switzerlad, 1998.

Publisher's Note: MDPI stays neutral with regard to jurisdictional claims in published maps and institutional affiliations.

(C) 2020 by the authors. Licensee MDPI, Basel, Switzerland. This article is an open access article distributed under the terms and conditions of the Creative Commons Attribution (CC BY) license (http://creativecommons.org/licenses/by/4.0/). 